\title{
LA-UR- $95=2321$ \\ Title: \\ INSTABILITIES IN FOIL IMPLOSIONS AND THE EFFECT ON RADIATION OUTPUT
}

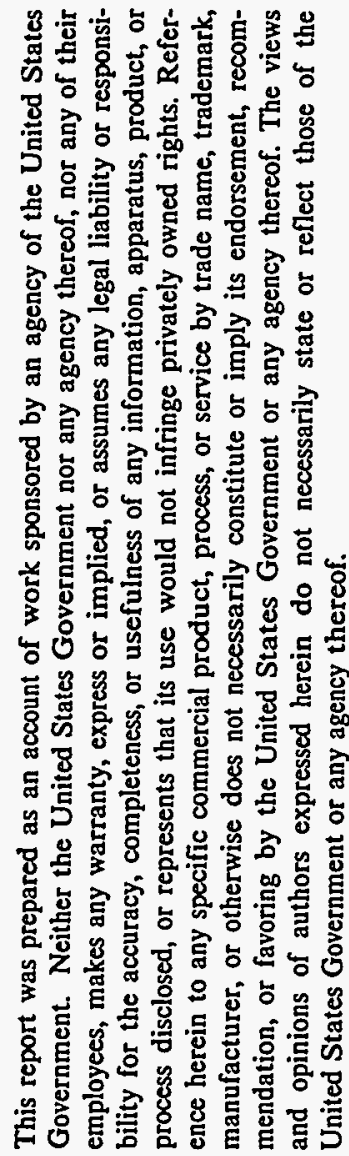

Submitted to:

H. Oona, D.L. Peterson, J.H. Goforth, M.L. Yapuncich, C. Findley, E. Lopez, J. Benage;-G. Idzorek, J.C. Cochrane, B. Anderson, J. Shlachter

10th IEEE International Pulsed Power Conference<smiles>CC(C)(C)C1CCCCC1</smiles>

DISTRIBUTION OF THIS DOCUMENT IS UNLIMITEQ

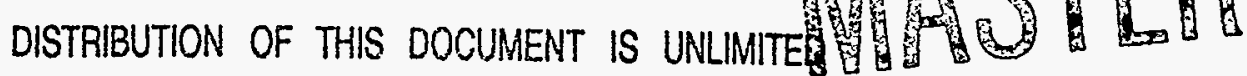

Los Alamos National Laboratory, an affirmative action/equal opportunity employer, is operated by the University of California for the U.S. Department of Energy under contract W-7405-ENG-36. By acceptance of this article, the publisher recognizes that the U.S. Government retains a nonexclusive, royalty-free license to publish or reproduce the published form of this contribution, or to allow others to do so, for U.S. Government purposes. The Los Alamos National Laboratory requests that the publisher identify this article as work performed under the auspices of the U.S. Department of Energy. 


\section{DISCLAIMER}

Portions of this document may be illegible in electronic image products. Images are produced from the best available original document. 


\title{
INSTABILITIES IN FOIL IMPLOSIONS AND THE EFFECT ON RADIATION OUTPUT
}

\author{
H. Oona, D.L Peterson, J.H. Goforth, M.L. Yapuncich, C. Findley, E. Lopez \\ J. Benage, G. Idzorek, J.C. Cochrane, B. Anderson, J. Shlacter \\ Los Alamos National Laboratory \\ Los Alamos, New Mexico, 87545 USA
}

Introduction

One of the aims of the Athena program at the Los Alamos National Laboratory is the generation of a high fluence of soft $x$-rays from the thermalization of an radially imploding foil. In the experiments in Athena program, a large axial current is passed through a cylindrical aluminum foil. Under the action of the Lorentz force, the resulting plasma accelerates toward the axis, thermalizes, and produces a fast soft x-ray pulse with a blackbody temperature up to several hundred electron volts. In order that there be the maximum power compression and the highest $\mathrm{x}$-ray fluence and temperature, the plasma stagnation on axis must occur very promptly. This requires that the imploding plasma be as thin and symmetric as possible. A serious problem in the thermalization process is the formation of instabilities in the plasma due to the self-magnetic field that governs the implosion of foil. A large diagnostic effort was developed to capture the details of the implosion and instability growth in several foil implosion experiments.

In this report, we will present visible light images and x-ray data designed to study the effects of foil mass, current, and initial perturbations on the instability growth during foil implosion. Representative data is presented from several experiments using the Pegasus capacitor bank system and the explosively driven Procyon system. These experiments are labeled Peg 25 and Peg 33 for the Pegasus experiments and PDD1, PDD2 and PRF0 for the Procyon experiments. In these experiments, all foils had radii of 5 centimeters but varied in mass and initial conditions. Experimental data from several shots were compared with each other and to a radiation magnetohydrodynamic (RMHD) computation and described in a separate paper. ${ }^{1}$. The data obtained from these experiments and the analysis has given us understanding for the physical mechanisms involved and insight for future experiments and has lead us to propose methods for minimizing the instability growth and maximizing the radiation output. In particular, we observed that wrinkles and other physical anomalies in the initial shape of foil do not appear to contribute to the growth of the instabilities.

\section{The Experiments}

Two systems used at LANL to supply the current to the imploding foil are the Pegasus II capacitor bank and the explosive flux compression generator of the Procyon system. The Procyon generator ${ }^{2}$ produces up to 18 mega Joules of energy, and from an initial current of 460 kilo Amperes, can supply 22 mega Amperes of current to a 73 nano Henry load. The Pegasus II capacitor bank ${ }^{3}$ is an excellent system for studying implosion physics. It is a 4.5 mega Joule system which supplies approximately 6 mega Amperes into the implosion load.

Diagnostic tools used in the experiments for studying the implosion physics and the generation of instabilities include visible framing cameras and $x$-ray imaging systems. High-speed visible light framing cameras (Imacons) were used to observe the implosion as the plasma converged and stagnated on axis. Due to the nature of the experiments, the visible images were transferred via mirrors from the experiment to a location where the cameras were protected. Computer enhancement was used study the details of the implosion from the images. Time resolved and time integrated $\mathrm{x}$-ray images and the total $\mathrm{x}$-ray output were obtained from bolometers and $\mathrm{x}$-ray diodes (XRD). The $\mathrm{X}$-ray imaging was done with the cameras attached to the vacuum chamber of the foil load. Previous experiments ${ }^{4}$ allowed us to develop protection for the $x$-ray systems so they could survive the shock and debris from high explosives in the Procyon experiments.

The thickness of the foil for the experiments were 0.8440 and 0.7380 microns for Peg 25 and Peg 33 respectively and 3.5770, 4.3090 and 4.0320 microns for PDD1, PDD2, and PRF0. In all experiments, the thin cylindrical foils were turned into plasma shells by the high current from either the Procyon explosive generator or the Pegasus capacitor bank. The shells imploded under the action of the high magnetic fields produced by the current.

Through the series in Figs. 1-3 of radial views from the visible light framing camera, we can observe the chronology of implosion, instability, and radiation production in Peg 25 and Peg 33. The images in Figs. 1 and 2 


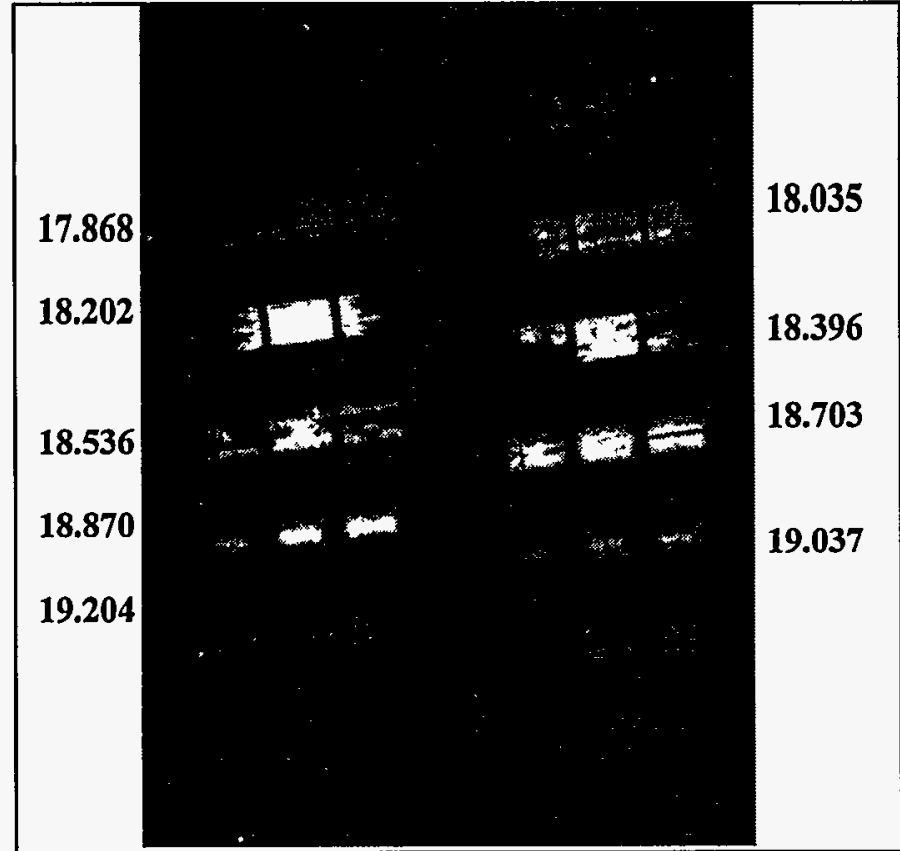

Fig. 1 Time sequence of Peg 25 images are shown. Times are given in microsecond and the interframe time is $167 \mathrm{~ns}$ The implosion on axis takes place at the frame labeled 18.536. After the implosion "Flaps" move from top and bottom and close off the area. have an inter-frame time of 167 nanoseconds with an exposure time is 32 nanoseconds. The image in Fig. 3 has an inter-frame time of 100 nanoseconds and an exposure time is 20 nanoseconds. Figures 8,9 , and 10 give similar images for PDD1, PDD2 and PRF0 respectively, all with interframe times of 167 nanoseconds.

The instabilities and their evolution in both the Pegasus and the Procyon experiments are clearly seen in the images. The common element in all these images is short wavelength mode instabilities that develops in the initial phase of the implosion. The instability evolution and the short wavelength instabilities appear very clearly in the amplified contour plot in Fig. 4 of the foil edge in the two Pegasus experiments. In time, the short wavelength modes saturate and form long wavelength mode instabilities that vary from experiment to experiment depending on foil mass and current. The long wavelength instabilities are the responsible for destroying the uniform prompt pinch on axis. As discussed in these proceedings ${ }^{1}$, the long wavelength instabilities influence the resulting current and voltage wave forms and the output $x$-ray

temperature and fluence.

Another, unexpected effect that seems to influence the x-ray radiation output is visible in all the images in Figs. 1-3 and 8-10. This is a "flap", a feature that moves in the axial direction from the top and bottom sides of the foil. This flap is observed in the images in both Pegasus and Procyon experiments. After the flap appears, it grows to block the view to the center of the implosion region and modifies the radiation output. The time of the initial appearance of this feature varies with experiment. In some experiments, it appears at pinch time and in others, it appears at much later. On Peg 33, in addition to the visible framing camera images, we were able to take time resolved $\mathrm{x}$-ray pictures which were filtered to observe different $\mathrm{x}$-ray energies. Fig. 5 shows a comparison of a visible framing camera image and an aluminum filtered $\mathrm{x}$-ray picture. These pictures were taken at almost identical times through adjacent ports approximately $12^{\circ}$ apart on the load chamber. Remarkably, the images look virtually the same, and the $\mathrm{x}$-ray pictures also show the flaps. Another $\mathrm{x}$-ray image taken with a carbon filter eliminates the view of the flaps. This observation and the fact that aluminum filters will transmit aluminum $x$-rays suggest that the flaps are due to radiation from cool aluminum plasma and may be related to wall effects on the growth of instabilities.

The effect of the flaps on x-ray production can be seen in the two Pegasus experiments. Experimental output and foil behavior were in good agreement with calculations in Peg 25. As shown in Fig 6, the calculated and measured current and bolometer power pulse agree extremely well. Experimentally, we measured 240 kilo Joules total fluence with unfiltered bolometers and 300 kilo Joules with XRDs on Peg 25; pre-shot calculations predicted 250 kilo Joules. On Peg 33, however, we measured only half as much radiation: 135 kilo Joules with bolometers and 175 kilo Joules with XRDs. Observations of the framing camera images, as seen in Figs. 2 and 3, reveal that the plasma stagnation on axis was much "cleaner" in Peg 25 than in Peg 33. In addition, the flaps, composed of colder foil/wall material, appeared after the pinch in Peg 25 but roughly at the time of the pinch in Peg 33. The maximum X-ray radiation appears in frame $18.536 \mu \mathrm{sec}$. in Peg 25 as compared with frames $17.5 \mu$ sec. in Peg 33 . We suspect the differences are due to the sloppier pinch in Peg 33 and the absorption of the radiation by the flaps. Elimination of the flaps may be possible by modifying the upper and lower implosion foil glide planes and by providing an exit for the cooler material. An upcoming experiment is designed to address this issue. 


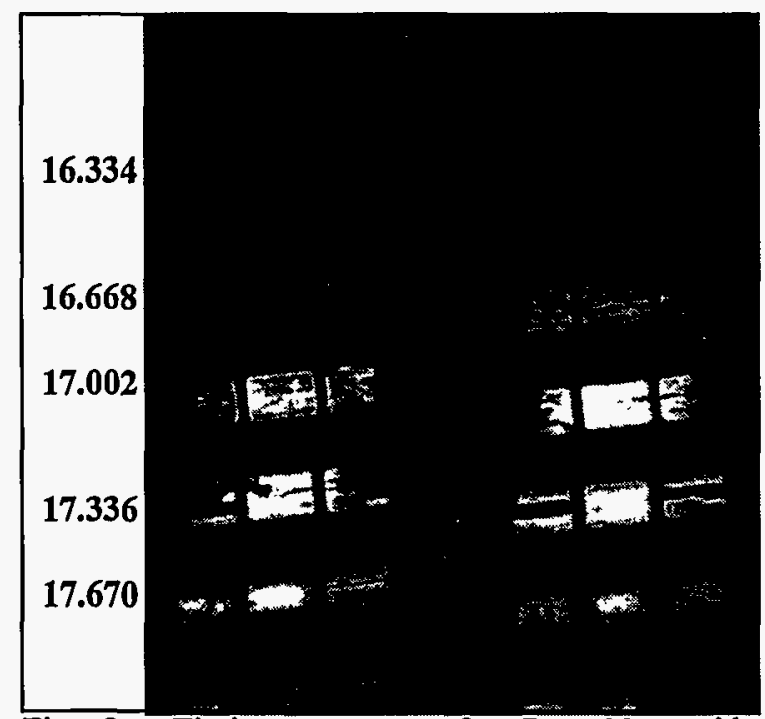

Fig. 2 Timing sequence for Peg 33 with interframe time of $167 \mathrm{~ns}$. X-ray radiation was produced at 17.5 microseconds. Large instabilities prior to pinch are seen, and the "flaps" have progressed significantly more than in Peg 25 .

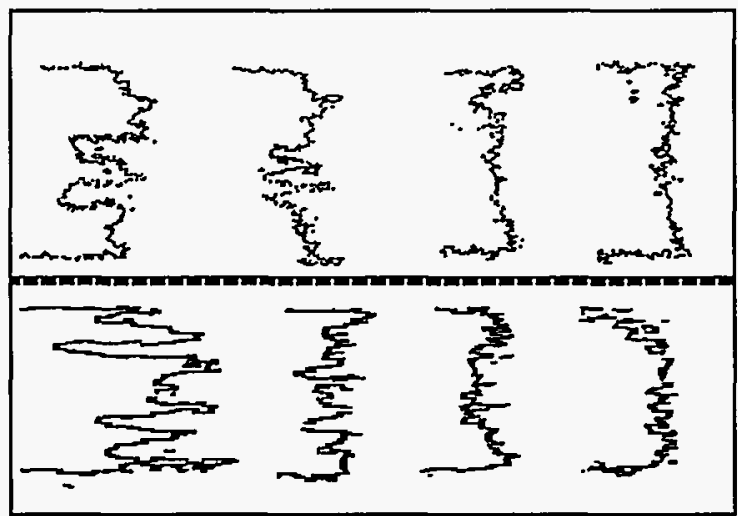

Fig.4 Foil initiation from separate experiments. The features of the foil edge are amplified horizontally. Total time observed is $800 \mathrm{~ns}$. All experiments show the same early instabilities.

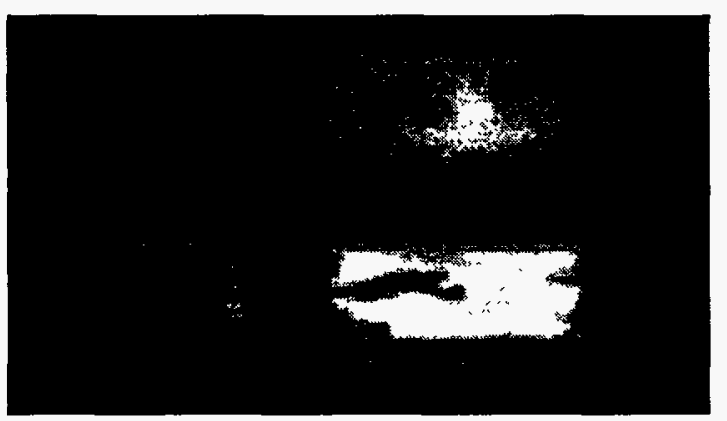

Fig 6 Comparison of aluminum filtered x-ray image (top) and a visible framing image (bottom). Similarities suggest the same regions for $\mathrm{x}$ rays and visible light. "Flaps" are seen in both.

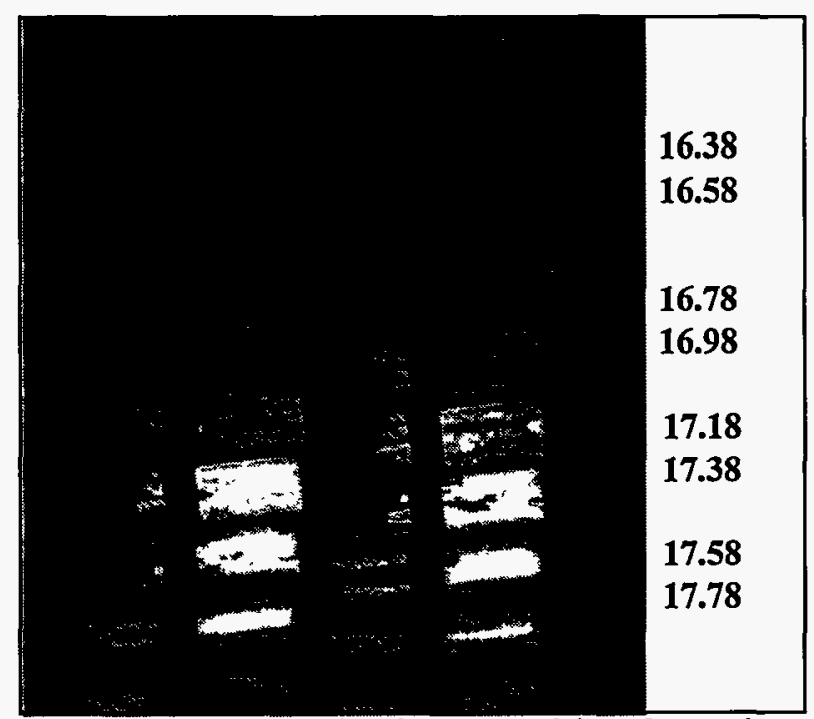

Fig. 3 Higher resolution images and interframe time of 100 ns.on experiment Peg 33. View is from a different angle. Details of foil initiation and mode evolution are clearly seen. Also, the initiation of "flaps" has become apparent.

Even though the main purpose of the framing images is to illuminate the plasma behavior at pinch time, we have observed some other unusual features in the plasma. These features, shown in Fig. 7, have appeared in all the experiments. The clearest images were obtained from Peg 33 . These late time features persist for several microseconds and might be related to blow off from the wall. A more interesting explanation is the arrangement of the atoms into domains similar to the magnetization and closure domains that occur in solid state magnetic materials.

Framing camera pictures for the high energy and high current Procyon experiments PDD1, PDD2, and PRF0 are shown in Figs. 8 - 10. Of these experiments, PDDl and PDD2 were physically identical. PRF0 was a modified experiment that had the upstream glide plane electrode that protruded into the implosion region. The glide plane electrode generated a high temperature plasma source by creating a plasma jet and forcing it through a hole in another downstream glide plane.

The images in Fig. 8 from PDDI appear weak and disappear completely at pinch time. We discovered later that the Pyrex entrance windows turn dark at the radiation fluence levels of these experiments. The entrance windows were changed to Quartz for PDD2 and PRF0. Also, due to the additional windows and mirrors that are used to transfer the image from the explosive firing table to the bunker, the Procyon images are not as sharp as the images from the Pegasus series. On the other hand, we notice that the amplitude of the instabilities in the Procyon foils are not quite as large as the instabilities in the Pegasus experiments. This may be due to the higher current and rate of change of current 


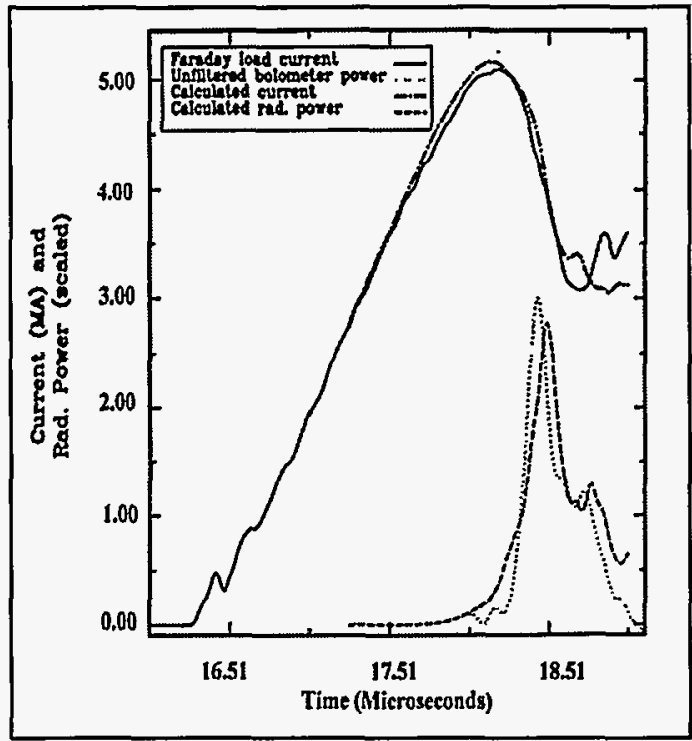

Fig. 6 Comparison of measured and calculated current for Peg 25. Calculated and measured bolometer power pulses are also shown. The total flunce was about 250 kilo Joules.

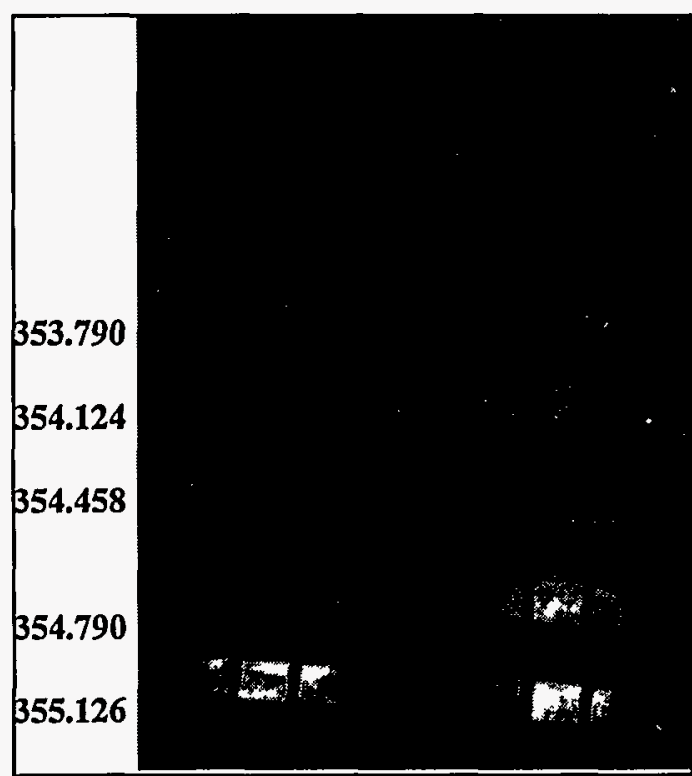

Fig. 8 Timing sequence for PDD1. Interframe time is $167 \mathrm{~ns}$. The pinch in this experiment occurred at 355.46 microseconds. Due to the radiation the Pyrex window turned dark Total of 1.5 mega Joules was produced.

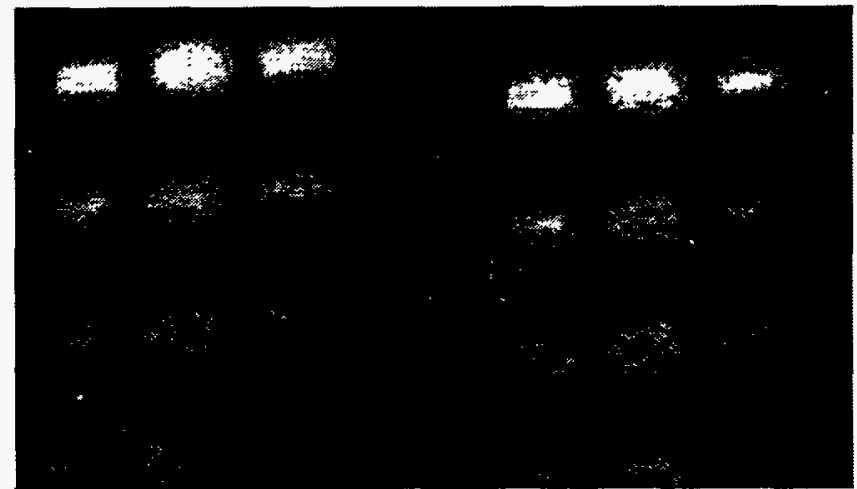

Fig. 7 This is a late time feature that appears in all experiments. It is mainly shown for curiosity. The total time shown covers 1.3 microsecond after the pinch. It may be related to orientation of the plasma atoms by the high magnetic field into magnetic domains.

in the Procyon experiments. Current profiles are shown in Fig 11. Additional discussion of these experiments is provided in another paper ${ }^{2}$ in these proceedings.

PDD1, the first experiment in the Procyon series, gave a total radiation output measured by bolometers and XRDs of 1.5 mega Joules. This results is in good agreement with the calculated estimate of 1.7 mega Joules. Unfortunately, in this experiment, we did not see the details of a continuous evolution of the instabilities to pinch time. In Fig. 8, pinch occurs at frame time 355.46 which is dark due to radiation darkening of the Pyrex window.

PDD2 images in Fig. 9 show the initiation of the foil and the presence of very large wrinkles. Careful observation of these images suggest that the wrinkles do not have an effect on instability growth. This experiment was electrically a more perfect experiment than PDD1 but it produced significantly less radiation. Bolometers measured 375 kilo Joules. XRDs located on the side opposite the bolometer measured 350 kilo Joules of total radiation. Another set of XRDs located at right angle to the bolometer gave a total radiation of 640 kilo Joules. This suggests a bad asymmetry or an off center implosion. The visible images show the implosion to be slightly off center. The $x$-ray pinhole cameras suggest that pinch was about $0.75-1.0$ centimeters from the axis. Since instabilities sometimes generate plasma bubbles that collapse, the radiation in this experiment might have been produced from a small spot of hot, collapsed plasma with the rest of the plasma remaining cold. Indications of such a scenario is shown in the second image in Fig 12.

The third Procyon experiment, PRF0, had a different purpose but it provided us with information on ways of modifying instability growth and generating a "clean" stagnation on axis. As in the PDD2 experiment, the PRF0 foil contained many initial wrinkles that are not related to the growth of instabilities. The images and instabilities, as shown in Fig. 10, evolved in the same way as they did in PDD2. However, at pinch time, the region that normally is full of plasma in earlier experiments appears in PRF0 as a clean, narrow pinch on axis. Modification of the glide plane electrode in this experiment forced the location of the pinch to be on axis, and the large hole at the 


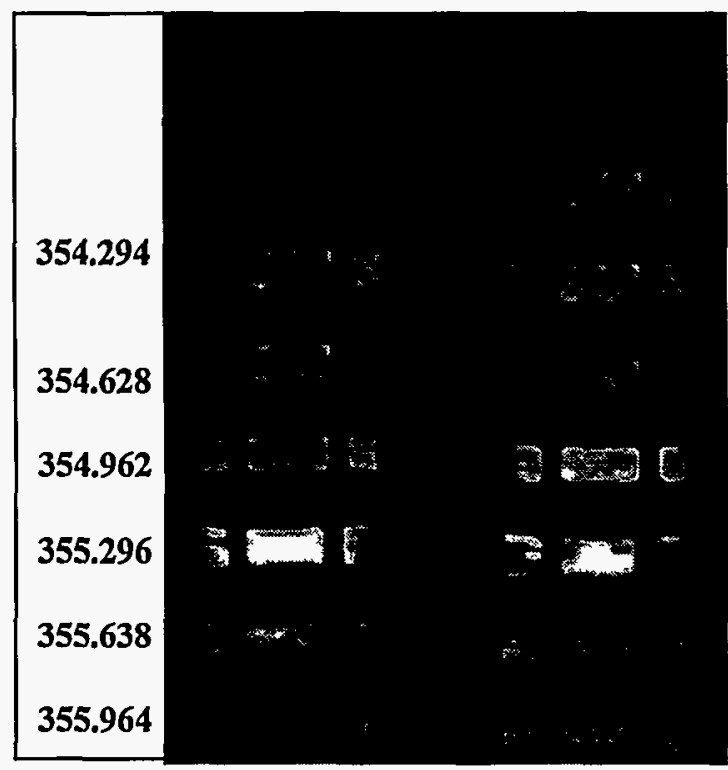

Fig. 9 Timing sequence for PDD2. Inerframe time is $167 \mathrm{~ns}$. The large wrinkles in the foil are clearly seen. Pinch time is at 355.63 and we notice the "flaps" beginning to obscure the center. Instabilities become noticeable at 355.96.

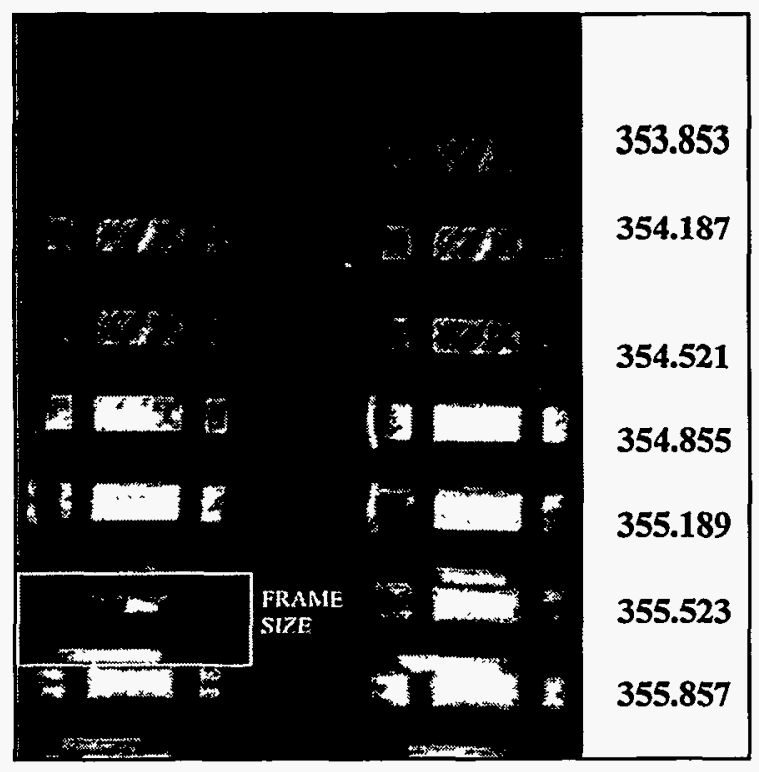

Fig. 10 Experiment PRF0. Large wrinkles are again apparent. The horizontal striations at 354.521 are instabilities due to the magnetic field. The box indicates pinch time and the extent of the frame. A jet is observed at the downstream electrode.

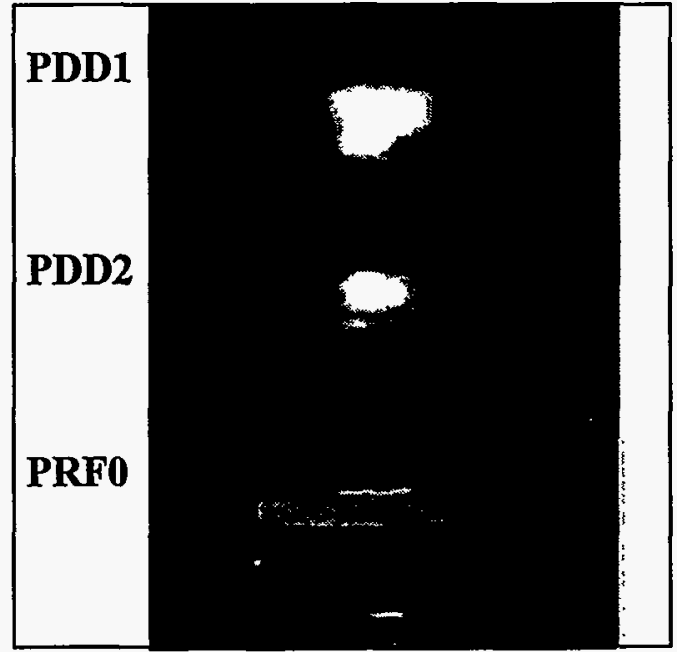

Fig. 12 Comparison of $\mathrm{x}$-ray pinhole camera images. The radiating source areas are very different for each experiment.

Fig. 11 The current from the generator and at the load are shown. The x-ray pulse from PDD1 is also shown. The electrical signals in all the Procyon experiments are remarkably reproducible. The curves labeled are: $\mathrm{A}=\mathrm{PRF} 0 ; \mathrm{B}=\mathrm{PDD} 1 ; \mathrm{C}=\mathrm{PDD} 2$.

downstream electrode helped "clean" out the implosion region. The radiation from this experiment was measure to be 640 kilo Joules with the bolometers and 580 kilo Joules with the XRDs.

Reasons for the different radiation fluences in PDD1, PDD2 and PRF0 are summarized by the $\mathrm{x}$-ray pinhole camera pictures in Fig 12. These images show from top to bottom PDD1, PDD2, and PRF0. Even though physically PDD1 and PDD2 are identical experiments, they show different radiating source sizes and hence different fluences. In addition, PRF0 shows a broad radiating source with a hot spot in the center. 


\section{Conclusions}

A series of experiments from the explosive Procyon system and the Pegasus capacitor bank has provided data for an understanding of radiation loss mechanisms during foil implosion. Our paper is composed mainly of self revealing series of foil implosion images that provide an understanding of the physical mechanisms involved. In experiments that showed lower than expected radiation fluences, the loss of radiation appears to be due to the absorption of radiation by cold material from the foil or the electrodes. The cold material can be due either to the way that foil instabilities distribute the mass at implosion time or to radiation "blow-off" from the electrodes. It appears that the majority of the cold material is due to the distribution of foil mass. Addition features present in the foil implosion images are "magnetohydrodynamic bubbles" which break at implosion time and provide a clear view to the implosion region. Visible light images and time resolved and filtered x-ray images also show "flaps" that develop from the top and bottom sides of the foil. From the images we have determined that the flaps are probably due to cold foil material. Initial foil behavior was similar in both the lower current Pegasus system and the more massive, higher current Procyon system At later time, however, the Procyon foils imploded more symmetrically and more quickly that the Pegasus foils. We also observed that the severe initial wrinkles in the foils (initial perturbations) do not seem to influence the initiation of the instabilities that grow.

A discussion of changes in the electrode geometry for the Procyon PRF0 experiment is explained in the text. We were able to obtain a sequence of pictures showing that in this experiment the load region was clear of "other plasma" at implosion time. Future modifications include electrode geometries that allow for the colder plasma to escape and minimize the effects of absorption. Foils made of different materials are also being considered to tune the system optimally for current and the rate of change of current.

We have successfully demonstrated that that we can generate 1.5 mega Joules of radiation and have learned how to fine tune the system to provide a high fluence and high temperature implosion by minimizing the presence of colder plasma in the load region. Future experiments will aim at reaching higher goals of multi-mega Joule and several hundred electron volt temperature radiation sources.

\section{References}

1. D. L. Peterson, et. al., "Comparison of 2-D Simulation Results with Experimental Results from the PEGII-25 Radiation Experiment on the Los Alamos Pegasus II Capacitor Bank," these proceedings.

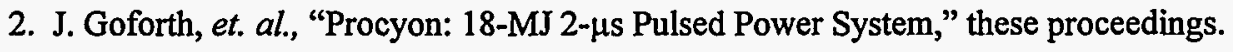

3. J. C. Cochrane, et. al., "Plasma Flow Switch and Foil Implosion Experiments on Pegasus II," Ninth IEEE International Pulsed Power Conference, Albuquerque, NM, June 21-23, 1993, pp. 805-807.

4. H. Oona, et. al., "Plasma and Electrical Diagnostics for Procyon Experiments," Eighth IEEE International Pulsed Power Conference, San Diego, CA, June 16-19, 1991, pp. 633-636. 